\title{
Cross-fringe versus single-fringe probabilities
}

\author{
W. Qin ${ }^{1}$, P. Fraundorf ${ }^{2}$ \\ ${ }^{1}$ Advanced Products R\&D Lab, Freescale Semiconductor, Inc., MD CH305, 1300 N. Alma School Road, \\ Chandler, AZ 85224 \\ ${ }^{2}$ Department of Physics and Astronomy and Center for Molecular Electronics, University of Missouri-St. \\ Louis, St. Louis, MO 63121
}

High-resolution TEM is well-suited to characterizing nanocrystals, where lattice fringes serve as a source of structural information $[1,2]$. Based on 2D lattice fringe images taken at different specimen orientations, 3D lattice parameters can be determined [3-6]. Recent work has shown that lattice fringe-visibility maps, a thinspecimen extension of bend-contour and channeling-pattern maps, can assist crystallographic study in direct space much as do Kikuchi maps in reciprocal space [7]. A nanocrystal can be tilted while the condition for visualizing a set of lattice fringes is maintained so as to "acquire" new lattice fringe normals (co-vectors), and thus continually refine a basis triplet containing information on both the nanocrystal's lattice and its orientation. Local specimen thickness measurements are another promising possibility.

Probabilities inferred from fringe-visibility maps further allow one to quantify the abundance of fringes in collections of randomly-oriented nanoparticles. In the thin-specimen limit, a fringe-visibility band has a band-width proportional to $d / t$, rather than the $\lambda / d$ proportionality expected for large $t$, where $d$ is the lattice spacing, $t$ is specimen thickness, and $\lambda$ is electron wavelength. This follows from the expression for bandwidth half-angle at arbitrary thickness:

$$
\alpha_{\max }=\sin ^{-1}\left[\frac{d f}{t}+\frac{\lambda}{2 d}\left(1-\left(\frac{d f}{t}\right)^{2}\right)\right],
$$

where $\mathrm{f}$ is a "visibility factor" on the order of 1 that empirically accounts for signal-to-noise in the method used to detect fringes [7]. The first term in (1) dominates for $t<2 d^{2} f / \lambda$ and therefore in typical TEMs for inter-atom spacings in particles $10 \mathrm{~nm}$ in size and smaller.

Fig. 1 shows the $\{200\},\{111\}$ and $\{220\}$ fringe-visibility bands of a spherical f.c.c. nanocrystal. The probability of the (hkl) lattice plane to show is therefore that fraction of the solid angle subtended by the corresponding visibility band, i.e. $\mathrm{p}_{(\mathrm{hkl})}=\sin \left[\alpha_{\max }\right] \cong \mathrm{d}_{\mathrm{hkl}} \mathrm{f} / \mathrm{t}$ in the thin-specimen limit. Band intersections correspond to regions of visible cross-fringes. Calculation of the area of an intersection between visibility bands [7] indicates that flat-polygon intersection areas are an excellent small-angle approximation, in some cases with errors on the order of $\alpha_{\max }{ }^{6}$. With this approximation, the probability of cross-fringes from lattice planes 1 and 2, whose fringe-visibility bands have half-widths $\alpha_{1}$ and $\alpha_{2}$ and intersect at an angle of $\phi$, is $p_{1 \times 2}$ $=2 \alpha_{1} \alpha_{2} /(\pi \sin \phi)$.

Figure 2 illustrates the fraction of randomly-oriented fcc particles showing only un-crossed (111) fringes, and that showing $<110>$ zone cross-fringes. It is obvious that cross-fringe grains become more abundant than single-fringe grains as the grain diameter $t$ decreases below $3 \mathrm{~nm}$. This is because the zone area increases as $(\mathrm{df} / \mathrm{t})^{2}$ while the single-fringe region increases as $(\mathrm{df} / \mathrm{t})$ and decrease in length at the expense of the zones. This model suggests, moreover, that the crossover size is quite sensitive to the visibility factor $\mathrm{f}$ for a given microscope/specimen combination. In such a small size range, though, the broadening of reciprocal spots warrants caution, as deceptive lattice fringes that are "Moires" instead of direct representations of the lattice planes may be formed, as a result of the low-pass filtering of projected potentials by the microscope [2]. Figures 3 and 4 show how zone axis areas (overlaps of bands) change as smaller spacings become reliably visible.

References: 
1. S.-C.Y. Tsen, P. A. Crozier, J. Liu, Ultramicroscopy 98(2003)63-72.

2. J. O. Malm, M. O'keefe, Ultramicroscopy 68(1997)13.

3. P. Fraundorf, Ultramicroscopy 22(1987)225

4. P. Möck, Cryst. Res. Technol. 26, 653-658 (1991).

5. W. Qin and P. Fraundorf, Ultramicroscopy 94(2003)245-262

6. W. Qin, Ph. D. Thesis, UM-StL/Rolla, 2000

7. P. Fraundorf et al., Making sense of nanocrystal lattice fringes, arXiv:cond-mat/0212281 (2005).
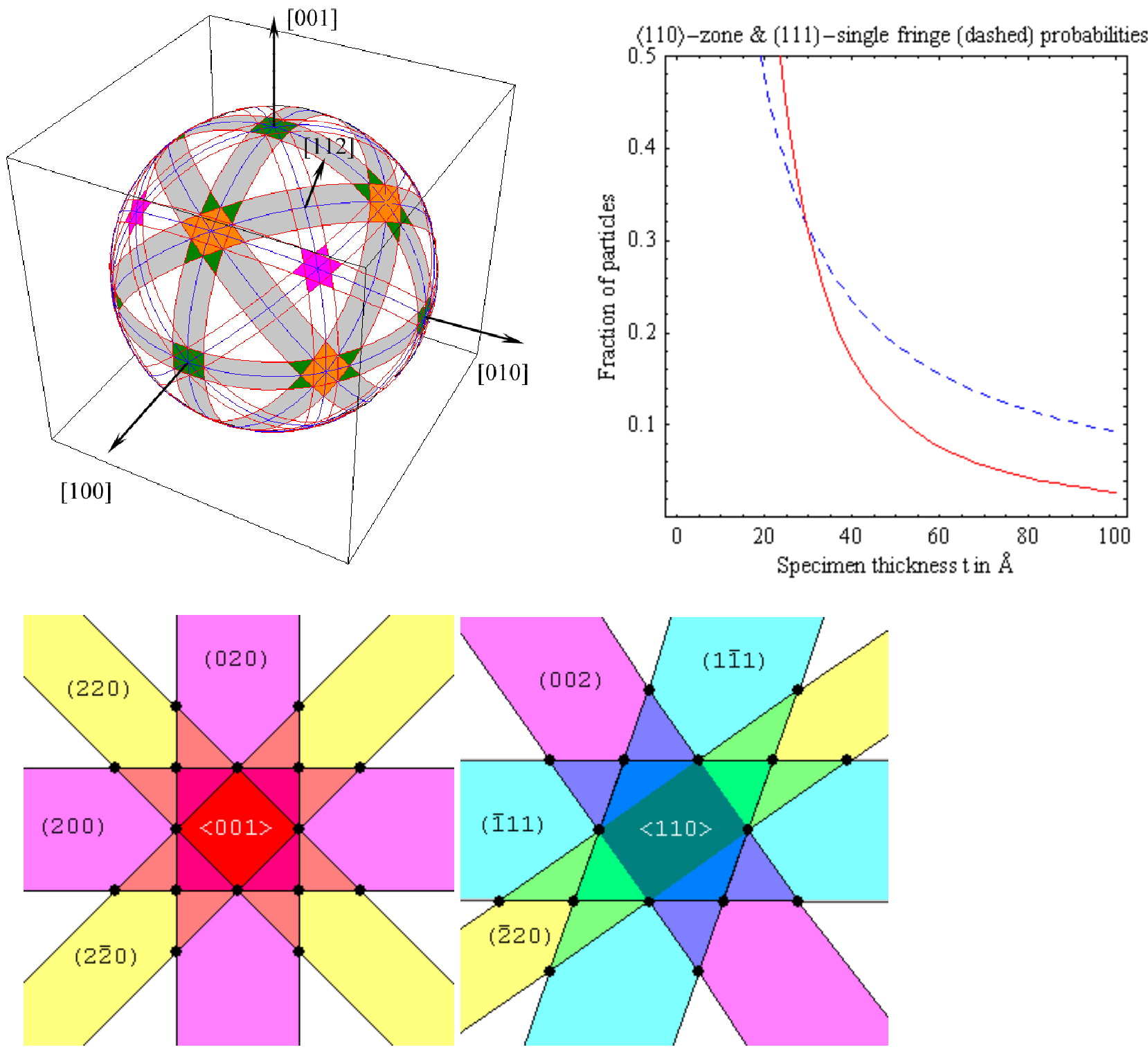

Figure 1 (upper left): The $\{200\},\{111\}$ (both shaded) and $\{220\}$ (not shaded) fringe-visibility bands of a spherical f.c.c. nanocrystal.

Figure 2 (upper right): The reversal in relative abundance for cross-fringe versus single-fringe particles as a function of particle diameter.

Figure 3 (lower left): Polygonal cross-fringe zones for fcc $\langle 001\rangle$ in the thin-specimen/small-angle approximations, built up as first $\{200\}$ and then $\{220\}$ fringes become visible.

Figure 4 (lower right). Polygonal cross-fringe zones for fcc $\langle 110\rangle$ in the thin-specimen/small-angle approximations. 